\title{
Preliminary Demonstration of an IonCCD as an Alternative Pixelated Anode for Direct MCP Readout in a Compact MS-Based Detector
}

\author{
Omar Hadjar, ${ }^{1}$ William K. Fowler, ${ }^{1}$ Gottfried Kibelka, ${ }^{1}$ William C. Schnute ${ }^{2}$ \\ ${ }^{1}$ OI Analytical/CMS Field Products, Pelham, AL 35124, USA \\ ${ }^{2}$ Dionex Corporation, Sunnyvale, CA, USA
}

\begin{abstract}
We report on the preliminary testing of a new position-sensitive detector (PSD) by combining a microchannel plate (MCP) and a charge-sensitive pixilated anode with a direct readout based on charge-coupled detector (CCD) technology, which will be referred to as IonCCD (Hadjar et al. J Am Soc Mass Spectrom 22(4):612-623, 2011; Johnson et al. J Am Soc Mass Spectrom 22(8):1388-1394, 2011; Hadjar et al. J Am Soc Mass Spectrom 22(10):1872-1884, 2011). This work exploits the recently discovered electron detection capability of the IonCCD (Hadjar et al. J Am Soc Mass Spectrom 22 (4):612-623, 2011), allowing it to be used directly behind an MC. This MCP-lonCCD configuration potentially obviates the need for electro-optical ion detector systems (EOIDs), which typically feature a relatively difficult-to-implement $5-\mathrm{kV}$ power source as well as a phosphorus screen behind the MCP for conversion of electrons to photons prior to signal generation in a photosensitive CCD. Thus, the new system (MCP-IonCCD) has the potential to be smaller, simpler, more robust, and more cost efficient than EOID-based technologies in many applications. The use of the lonCCD as direct MCP readout anode, as opposed to its direct use as an ion detector, will benefit from the instant three-tofour-order-of-magnitude gain of the MCP with virtually no additional noise. The signal/noise gain can be used for either sensitivity or speed enhancement of the detector. The speed enhancement may motivate the development of faster lonCCD readout speeds (currently at $2.7 \mathrm{~ms}$ ) to achieve the $2 \mathrm{kHz}$ frame rate for which the IonCCD chip was designed, a must for transient signal applications. The presented detector exhibits clear potential not only as a trace analysis detector in scan-free mass spectrometry and electron spectroscopy but also as a compact detector for photon and particle imaging applications.
\end{abstract}

Key words: IonCCD, Pixelated anode, Microchannel plate, Direct MCP readout, Charged particle array detector, Proximity focus, Imaging

\section{Introduction}

Since their development in the 1960's, microchannel $\checkmark$ plates (MCPs) [4] have become a critical gain element in detection systems in many laboratories around the world. The versatility of the MCP makes it a gain-producing element of choice for energetic particles (ionic and neutral species), electrons, X-rays, and from UV to IR when used

Correspondence to: Omar Hadjar; e-mail: ohadjar@oico.com with appropriate photocathodes. The broad descriptive work of MCPs can be found in early work [5-16].

Being a two-dimensional amplifier with fast response $(<200 \mathrm{ps})$, high gain $\left(>10^{3}\right.$ to $10^{4}$ per plate), and high spatial resolution [ $150 \mathrm{lp} / \mathrm{mm}$ for Photonis (Sturbridge, MA, USA) $2 \mu$ pore size], an MCP is an effective charge multiplication element that preserves positional and temporal information. It is often used in conjunction with several types of coordinate encoding charge collection anodes with respective coordinate decoding electronics. Most popular anodes provide time and space information and are usually used for 
pulse counting in single particle and photon spectroscopy; they include two [17] and three-layer [18] delay-line anodes operating up to $1 \mathrm{MHz}$ count rate for true $3 \mathrm{D}$ imaging with certain multi-hit abilities $(<10)$. The delay-line method can also be used indirectly to read out PCB-based anode structures $[19,20]$. Analog-encoding anodes are of two varieties: (1) resistive anode encoder (RAE), which is often used together with 3 to $5 \mathrm{MCPs}$ to achieve maximum signalto-noise ratio, hence high resolution [21-23], and (2) discrete conductor segmented anodes, such as wedge-andstrip anodes [24]. For digital image production, both resistive and delay-line anodes are coupled to a constant fraction discriminator (CFD) for raw signal processing before being inputted to a time-to-digital converter (TDC).

In velocity mapping and interaction dynamics experiments where temporal resolution is a must, faster systems are needed such as delay-line detectors (DLDs) for true 3D imaging. Recently developed is the $1-\mathrm{kHz}$ optical-based complementary metal oxide sensor and field programmable gate array (CMOS+FPGA), which was designed for higher multi-hit $(>50)$ applications with online centroiding using the FPGA for real time imaging [25]. When time information is not critical, CCD camera readout is often used to access the optical signal from the phosphor screen (EOIDs) [26]. The IonCCD used in this work was described in detail elsewhere $[1,27]$. Briefly the semiconductor material used in regular CCD chips is replaced by a conductor material coupled to capacitors. The incoming charge is integrated by the capacitors, inducing a voltage change, which is function of the incoming charged particle flux and integration time. Each individual pixel output voltage is then amplified through a two-gain-value pre-amplifier $(G=1,2.2)$. The analog voltage is digitized by a 16-bit analog-to-digital converter. The clocking and voltage measurements are controlled by an FPGA, which also determines the order of the measurements, so that each signal voltage can be matched up with its corresponding pixel, to reconstruct a profile or a spectrum.

In this preliminary, proof-of-concept paper, we introduce the IonCCD acting as an MCP direct readout anode rather than as a stand-alone ion detector. In this mode, we demonstrate that the IonCCD benefits from the MCP's "instant" $10^{3}$ to $10^{4}$ gain with virtually no increase in overall system noise, which is dominated by the IonCCD intrinsic noise. In principle, this $\mathrm{S} / \mathrm{N}$ gain can be used for enhancement of either sensitivity or speed of the IonCCD detector (up to its designed operating limit of $2 \mathrm{kHz}$ ). The improved speed should allow the hybrid detector to be used in non-scanning sector-field instruments for shortlived transient signals. Although instrument resolution was less than expected, improvements in resolution will be pursued in future work. The detector described in this paper is mounted in the focal plane of a miniature sector-field Mattauch-Herzog geometry mass spectrometer (MH-MS), described elsewhere [3]. The signal used in this work is that from the challenging $\mathrm{Xe}^{2+}$ ion with an effective one-half mass unit separation between the isotope peaks.

\section{Experimental}

The prototype MCP-IonCCD assembly was built with a single MCP plate at proximity focus condition with about $250 \mu \mathrm{m}$ between the MCP output face and the IonCCD chip. For these proof-of-concept experiments, we used a standard Photonis $\mathrm{MCP}$ as a matter of convenience, even though its circular geometry was inconsistent with the rectangular geometry of the IonCCD. This MCP is specified by the manufacturer as MCP 25/12/10/12 D 60:1, where, from left to right, $25 \mathrm{~mm}$ is the minimum quality diameter, $12 \mu \mathrm{m}$ is the channel center-tocenter spacing, $10 \mu \mathrm{m}$ is the channel diameter, $12^{\circ}$ is the channel bias angle with respect to the MCP normal, D stands for detection grade (lowest), and 60:1 is the aspect ratio MCP thickness to channel diameter. The geometrical detection efficiency or the open area ratio (OAR) of the MCP is $65 \%$ with an MCP thickness of $610 \mu \mathrm{m}$. Finally the depth of the nickel-based electrode penetration into the channel from the entrance and exit faces ('endspoiling') is twice the channel diameter $(\sim 20 \mu \mathrm{m})$. It has been calculated that the electrode endspoiling has an important influence on the output energy and angular distributions of electrons, (EDOE) and (ADOE), respectively [11].

The IonCCD was described in detail elsewhere [1, 27]. Briefly, it is a $51 \mathrm{~mm}$-long array of 2126 pixels, each being $21 \mu \mathrm{m}$ wide and $1.5 \mathrm{~mm}$ long, with a $3 \mu \mathrm{m}$ gap between them, producing a $24 \mu \mathrm{m}$ pitch and about $88 \%$ pixel area ratio (PAR). The pixel material was modified from a photosensing material for electron-hole pair creation to a conductive material coupled to a capacitor for charge collection and voltage conversion. The individual analog voltages are serially read over $2.7 \mathrm{~ms}$ or longer and digitized by a 16 bit analog-to-digital converter (ADC). IonCCD signals are commonly expressed as multiples of a digital signal concept called a "digital number" (dN). The $\mathrm{dN}$ represents a single step in the ADC's output, and it was quantified in earlier work [1] as the detector's response to 100 ions. The integration time can be tuned from $83 \mu \mathrm{s}$ to $5 \mathrm{~s}$ to avoid saturation at high fluxes and to increase sensitivity at low fluxes. Using minimal readout and integration times of 2.7 and $0.1 \mathrm{~ms}$, respectively, (the readout time is limited by the actual camera electronics) the IonCCD produces 357 mass spectra per second, i.e., it operates at a $357-\mathrm{Hz}$ frame rate, although under these extreme conditions, the instrument's duty cycle is quite poor $(3.6 \%)$.

The IonCCD has random RMS noise amplitude of about $9 \mathrm{dN}$ at sub-100-ms integration times; this translates to 900 vacuum electrons RMS per pixel. The MCP's random noise contribution, when calculated as the square root of the dark current of the MCP as specified by the manufacturer $\left(0.5 \mathrm{pA} / \mathrm{cm}^{2}\right)$ and when normalized to the pixel area and a 1 -ms integration time, is 1 vacuum electron RMS, or roughly $0.1 \%$ of the IonCCD's noise contribution. Although this percentage goes up slightly as the integration time increases (e.g., $1 \%$ at $100-\mathrm{ms}$ integration time), it remains an insignificant fraction of the IonCCD's noise for most meaningful applications and integration times. Note also 
that because the IonCCD typically integrates many individual ion arrival events within a single integration period, the shot noise associated with the MCP's amplification of the individual ions is effectively averaged out by the IonCCD. This is a major strategic inducement for pairing the MCP with the IonCCD.

The experimental MCP-IonCCD is sketched in Figure 1 showing the respective key geometries of the MCP and IonCCD and the voltages that were used. The MCP holder was designed of PEEK material allowing proximity focus conditions between the circular MCP and the rectangular pixelated anode. The PEEK holder ensured an MCP-IonCCD air gap of about $250 \mu \mathrm{m}$, assuming the IonCCD chip was flush with the printed circuit board surface (i.e., the CCD board). The assembly was mounted on the focal plane of the MH-MS, which was described in detail in an earlier work [3].
To conduct the measurements, we used Xenon gas sample from a Matheson purity $99.995 \%$ cylinder (Matheson tri-Gas, Basking Ridge, NJ, USA), injected into a vacuum chamber from an atmospheric-pressure source through a highconductance-limit fused-silica capillary $(20 \mathrm{~cm}$ long by 50 um ID). The gas-phase compounds were ionized by means of resistively heated filament-induced thermionic electrons in an electron-impact ionization mode. The thermal ions were extracted and accelerated towards the analyzer entrance, which was a $100 \mu \mathrm{m}$ wide slit plate, and after passing through a well defined field-free region, the ions were curved $31.8^{\circ}$ through a $50 \mathrm{~mm}$ radius electro-static analyzer (ESA) and finally curved $90^{\circ}$ in the opposite direction in the permanent 1-T magnetic sector. This geometry allowed a double focusing of the dispersed ions in a single plane (focal plane). The peak shape of the focused $\mathrm{m} / \mathrm{z}$ ion was of a Gaussian distribution with a

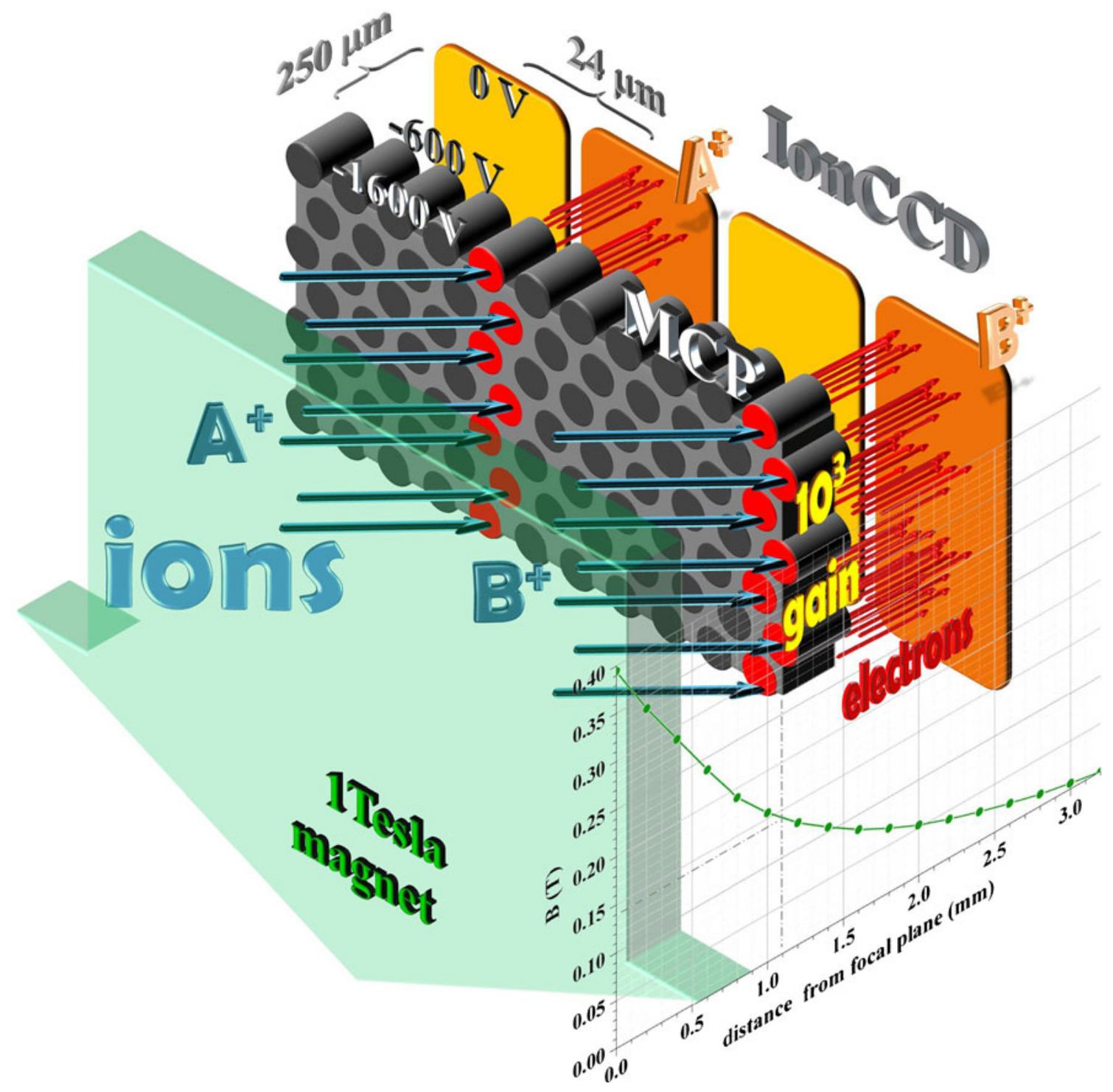

Figure 1. Schematic representation of a position-sensitive detector (PSD) consisting of a single microchannel plate (MCP) and an lonCCD type anode for direct signal readout. The MCP-lonCCD system is located at the outer fringe of the rapidly decreasing B-field of the sector-field type mass spectrometer 
FWHM proportional to the slit width and magnetic radius, and inversely proportional to the electric radius. The geometry of the analyzer allowed for a resolving power of $\mathrm{m} / \Delta \mathrm{m}=125$ at $10 \%$ valley definition ( $5 \%$ of the isolated peak height). To maintain the same spatial focusing of the instrument when using both detectors, the IonCCD and the MCP-IonCCD were mounted at roughly the same distance $(1.1 \mathrm{~mm})$ from the magnet exit face.

This system proved to be effective for characterizing the MCP-IonCCD as it provided sharp distributions with closely spaced peaks.

\section{Results and Discussion}

Due to the present design of the IonCCD with its protruding wire bonds, the circular MCP was chosen to have a diameter smaller than the IonCCD array length. Thus, the assembly holder was specially designed to drop the MCP down between the IonCCD wire bonds to achieve proximity focus conditions. This in turn limited the choice of the MCP outside dimension to a $32.7 \mathrm{~mm}$ diameter. With this limitation, the new detector focal length was given by the MCP effective diameter of $25 \mathrm{~mm}$, or half the focal length of the bare IonCCD. Clearly, therefore, the choice to insert a standard circular MCP between the wire bonds of the rectangular IonCCD significantly limited our mass range. Consequently, at acceleration voltage of $1.2 \mathrm{kV}$ and a magnetic field strength of $1 \mathrm{~T}$, singly charged Xe ions fell outside the working mass range and, thus, were not detected. Hence, the assembly characterization was performed with the doubly ionized species $\mathrm{Xe}^{2+}$. This allowed us to benefit from higher impact energies on the MCP $(2.4 \mathrm{keV})$, but at the same time it challenged the instrument's resolution with half-mass-unit $(0.5 \mathrm{u})$ separation at a nominal mass of $\sim 66 \mathrm{u}$. The positively charged ions produced by a floating source and MH-MS permitted the operation of the IonCCD at ground potential while allowing the MCP bias and outgoing electron acceleration to be varied, thus keeping the ion impact energy on the MCP constant. The short MCPIonCCD spacing allowed us to use a relatively low voltage gradient while ensuring high electric fields for the outgoing electrons. In early work [3], we demonstrated that the IonCCD can be floated at high voltages $( \pm 3 \mathrm{kV})$ which would facilitate its use as an MCP anode in different configurations, especially when ion sources and/or analyzers cannot be biased at high voltage. Thus, the floating capability of the IonCCD provides an extra degree of freedom for instrument design.

The illustrated magnetic field plot in Figure 1 was produced experimentally by probing the magnetic field outside the magnet, i.e., beyond the exit of the magnetic face. The curve shows the rapid drop of the B-field from the magnet exit face towards the MCP-IonCCD detector assembly. The MCP in this case was operated below $1400 \mathrm{G}(0.14 \mathrm{~T})$ with the B-field lines virtually perpendicular to the MCP microchannels. When using the IonCCD directly for detection, the ion source was floated to $1.2 \mathrm{kV}$, with the MH-MS and IonCCD at $0 \mathrm{~V}$. To maintain the same magnetic ion path and, hence, analyzer overall transmission, the ion source was floated to $-400 \mathrm{~V}$, with the MH-MS and front MCP at $-1600 \mathrm{~V}$, back MCP at $-580 \mathrm{~V}$, and IonCCD at $0 \mathrm{~V}$.

The isotopic natural abundances of the doubly charged Xe ions $[\mathrm{u}=128(1.92 \%), 129$ (26.44\%), 130 (4.08\%), 131 (21.18\%), 132 (26.89\%), 134 (10.44\%), and $136(8.87 \%)]$ are reflected in the spectrum in Figure 2a, which was generated while using the IonCCD for direct ion detection. For this experiment, the capillary used to introduce the Xe sample to the ion source was removed from the ionization volume and leaked directly to the vacuum chamber. This step caused the detected signal to be about 20 -fold lower than when the capillary was inserted into the ionization volume. This was necessary to ensure that the pressure in the vacuum chamber, as read by the system's vacuum gauge, was the same as the pressure in the ionization volume. It also served to dilute the sample when using the more sensitive detector (MCP-IonCCD). Moreover, although the IonCCD has been found to detect negative ions and electrons as well as positive ions, we have found that in its current design, its linear dynamic range is limited to two orders of magnitude in negative mode. This factor, too, affected our choice of experimental conditions for getting all of the most prominent $\mathrm{Xe}$ ions on scale, and within the linear range, as shown in Figure 2. Note in Figure 2 that because of our choice to get the most abundant Xe ions within the system's linear range, the two lightest $\mathrm{Xe}$ isotopes $(\mathrm{A}=124,126)$, each with $0.09 \%$ natural abundance, were not detected above the IonCCD noise.

But there is no reason in principle why the IonCCD cannot be redesigned to be a true bipolar detector with equivalent thousand-fold linear dynamic ranges in either polarity. Hence, plans have been made to incorporate this capability in future versions of this detector chip. We should note at this point that an IonCCD with a linear dynamic range of $10^{3}$ [1] would be a good fit to an MCP with a similar dynamic range. In fact, we calculated a linear dynamic range of slightly greater than $10^{3}$ for the MCP, given the manufacturer-specified maximum linear output of $0.8 \mathrm{uA}$ for the total effective surface $\left(4.9 \mathrm{~cm}^{2}\right)$ of the MCP, in conjunction with our known $\mathrm{m} / \mathrm{z}$ focusing conditions (our system focuses the ion beam to an area of $3 \times 10^{-4} \mathrm{~cm}^{2}$ ).

The table in Figure 2a shows the isotope peak coordinates on the IonCCD and the FWHM of the peaks expressed in numbers of pixels. The peak area $\mathrm{S}$ of the heaviest isotope $(136 \mathrm{u}$ ), expressed in dN as shown in Figure 2, was used to quantify the signal boost when comparing the signal from the IonCCD and MCP-IonCCD. The ion source settings, predominantly the ionization volume pressure and the ionizing electron beam current, were used to normalize the detected signal. The normalized signal $S^{*}=S /\left(P \bullet I_{e} \bullet t\right)$ is expressed in $\mathrm{dN} /(\mu$ Torr $\bullet \mu \mathrm{A} \cdot \mathrm{ms})$, where $\mathrm{P}$ is the ionization volume pressure in Torr, $I_{e}$ is the electron beam current in 


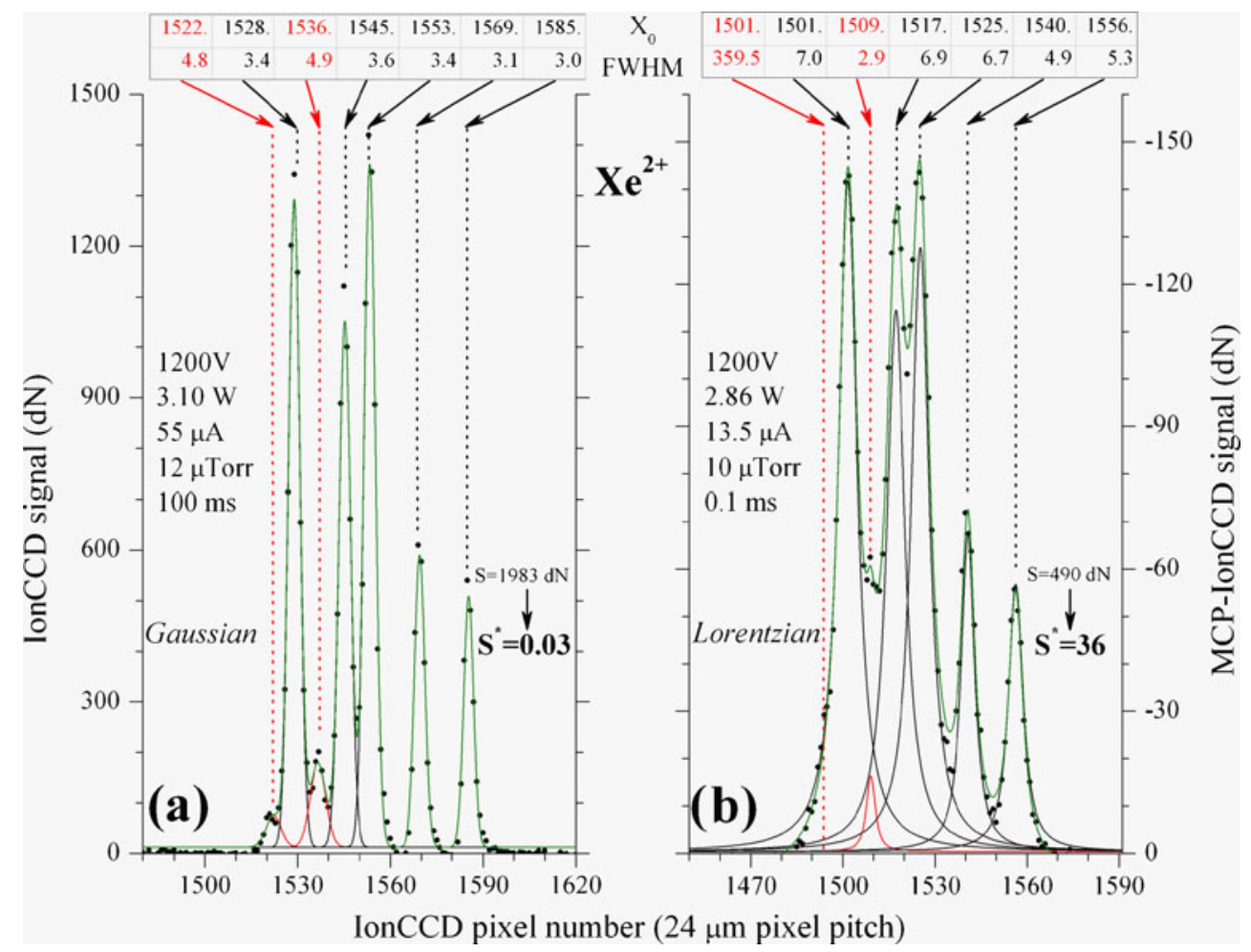

Figure 2. Isotopic abundance spectrum of $\mathrm{Xe}^{2+}$ using a miniature non-scanning sector-field mass spectrometer: (a) Using the IonCCD as a focal plane detector for direct ion detection and (b) Using the lonCCD as a pixelated anode to read out a microchannel plate (MCP). Note the normalized signal $\left(\mathrm{S}^{*}\right)$ enhancement of about $10^{3}$ given by the MCP when operated at $1000 \mathrm{~V}$ (see text). The tabulated information at the top of each figure contains the peak centroid location $\left(X_{0}\right)$ and the peak width (FWHM) of each peak in pixel numbers

$\mu \mathrm{A}$, and $\mathrm{t}$ is the integration time in ms. The peak widths given by the IonCCD (Figure 2a) are a realistic measure of the ion beam spatial focusing produced by the MH-MS. In fact, we demonstrated in early work [1] (Supplemental Information) that the IonCCD preserves the positional information of the ion impact, making it suitable as a beam profiler [2]. Figure $2 \mathrm{~b}$ shows the same experimental conditions as in Figure 2a but after replacing the IonCCD by the MCP-IonCCD. The shift of the isotopic distribution towards higher pixel number of about 30 pixels or $0.72 \mathrm{~mm}$ was due to a slightly higher ESA electric field than in the IonCCD experiment. Note the systematic broadening of the isotope peaks with a change from a Gaussian to a pseudoLorentzian distribution.

As shown in Figure 2, the peak width of ${ }^{136} \mathrm{Xe}^{2+}$ increased from 3 to 5.3 pixels when using the MCP as a gain element, an increase of about $70 \%$. In principle, one might expect the electrons streaming from the MCP's outlet face to be broadened or dispersed either by mutual coulomb repulsion or by any natural tendency of the electrons to leave the MCP going in different directions. But the electron extraction field was increased from 600 to $900 \mathrm{~V}$ with no resolution improvement, and the resolution displayed rapid degradation at lower extraction voltages. In addition, reducing the gain of the MCP, so as to decrease the density of electrons leaving the MCP for the IonCCD and thereby reduce Coulomb repulsions, also failed to improve the peak width. Thus, the causes of the peak broadening are, as yet, not understood. Although the observed degree of peak broadening represents a serious limitation for many potential applications, these first results were nonetheless considered very encouraging, given the rudimentary nature of this first attempt to pair these two key components.

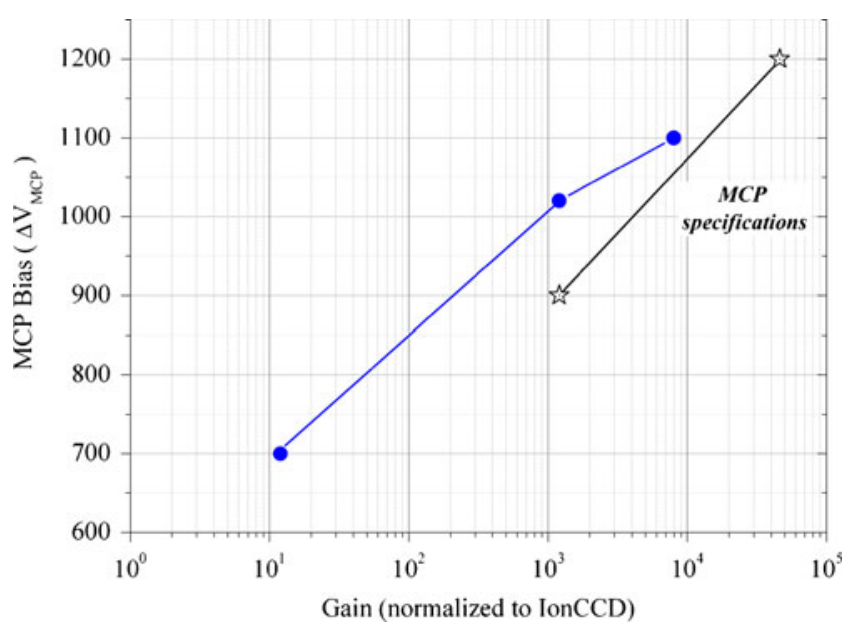

Figure 3. IonCCD signal readout as function of the voltage bias across the MC. The MCP manufacturer's specifications are indicated with stars 
As Figure 2 indicates, a signal boost of more than three orders of magnitude was recorded with the MCP operating at $1000 \mathrm{~V}$ bias voltage. This gain, which is the ratio between the two experimentally measured signals (after normalizing and averaging at least 100 consecutive frames), i.e., $\mathrm{S}^{*}$ $(\mathrm{MCP}-\mathrm{IonCCD}) / \mathrm{S}^{*}(\mathrm{IonCCD})=36 / 0.03=1200$, is quite close to the expected gain based on manufacturer's specifications. With such a gain, one can either use the IonCCD at high frame rates $(360 \mathrm{~Hz})$ at moderate sensitivities, or at lower frame rates for trace analysis, or with low ion beam current source output. This successful first-use of the MCP as a gain element will motivate the development of the IonCCD camera electronics to match the IonCCD chip's true speed of a $2 \mathrm{kHz}$ frame rate. Such speed, together with the MCP gain contribution, may lead to a very fast miniature mass spectrometer for transient fast processing and possibly for trace analysis applications as well.

To study the MCP gain, the voltage across the MCP was varied from 700 to $1100 \mathrm{~V}$, while keeping the $\mathrm{Xe}^{2+}$ impact energy and outgoing electron extraction field constant. The results are shown in Figure 3, where the manufacturer's MCP gain specifications are also shown in star symbols. The deviation of the experimental values from the manufacturer's specifications is to be expected when the MCP is operated in the transversal mode with respect to the B-field. The deviation is further increased due to the $65 \%$ open area ratio of the used MCP, which was not taken into account. Figure 1 shows that the B-field decreases rapidly to $<1500 \mathrm{G}$ at the MCP location. This result is very encouraging knowing that the measured B-field shown in Figure 1 is transverse to the microchannel axis of the MCP, a worst case scenario for MCP performance [6].

\section{Conclusion}

The MCP-IonCCD as used in this work exhibited clear potential for eventual use in MH-MS as applied to trace analysis and to $\mathrm{kHz}$-regime mass spectrometry for transient process studies. The MCP-IonCCD system demonstrated greater than a $10^{3}$ gain in signal with little or no increase in noise over that observed from the IonCCD alone. Moreover, there is no reason in principle why even higher gains cannot be achieved, as suggested by the experimental data of Figure 3. Though the present system is limited by its $2.7 \mathrm{~ms}$ readout time electronics, the success of the experiment reported here will further motivate the development of faster readout electronics to match the $2 \mathrm{kHz}$ capability of the IonCCD chip. Moreover, it may lead not only to useful onedimensional detectors for the MH-MS but possibly also to two-dimensional detectors for imaging applications lying well outside the field of mass spectrometry.

The observed loss of resolution was a seriously limiting issue that will have to be addressed in future development efforts. For example, we feel that there is room for improvement in both the choice of MCP and in the design of the IonCCD to facilitate its integration with the MC In addition, the limited dynamic range of the present IonCCD for negatively charged particles will dictate further development to produce a single chip that is capable of true bipolar operation.

The MCP in conjunction with the IonCCD (MCPIonCCD) in a mass spectrometer should be especially useful for GC/MS work, where narrow peaks may be observed and where low limits of detection may be required. It should be valuable as well for determining nonvolatile compounds in HPLC and related liquid-phase separations, which employ atmospheric pressure electrospray ionization sources (ESI) and other sources that are known to produce much less ion throughput compared to electron-impact-based ion sources. In addition, this technology may also prove to be of value in extending the $\mathrm{MH}$-mass spectrometer instrument into secondary ion mass spectrometry (SIMS). But many other potential applications for this technology exist as well. For example, to cite a few of the most recent and relevant publications in mass spectrometry, this approach may be beneficial for laser ablation miniature mass spectrometry (LA-MMS) for space application [28] as well as for the concept instrument based on distance-of-flight mass spectrometry (DOF-MS) [29].

\section{Acknowledgments}

The authors acknowledge the support of OI Analytical for this research. They thank CMS Field Products and Dionex Corporation for technical support. They also thank William Netolicky and Dr. Stephen M. Ritzau from Photonis for their valuable MCP-related feedbacks. The work was performed at CMS Field Products, a subsidiary of OI Analytical.

\section{References}

1. Hadjar, O., Johnson, G., Laskin, J., Kibelka, G., Shill, S., Kuhn, K., Cameron, C., Kassan, S.: IonCCD ${ }^{\mathrm{TM}}$ for Direct Position-Sensitive Charged-Particle Detection: from Electrons and $\mathrm{keV}$ Ions to Hyperthermal Biomolecular Ions. Journal of the American Society for Mass Spectrometry 22(4), 612-623 (2011)

2. Johnson, G.E., Hadjar, O., Laskin, J.: Characterization of the Ion Beam Focusing in a Mass Spectrometer Using an IonCCD ${ }^{\mathrm{TM}}$ Detector. Journal of the American Society for Mass Spectrometry 22(8), 13881394 (2011)

3. Hadjar, O., Schlatholter, T., Davila, S., Catledge, S.A., Kuhn, K., Kassan, S., Kibelka, G., Cameron, C., Verbeck, G.F.: IonCCD Detector for Miniature Sector-Field Mass Spectrometer: Investigation of Peak Shape and Detector Surface Artifacts Induced by keV Ion Detection. Journal of the American Society for Mass Spectrometry 22(10), 18721884 (2011)

4. Wisa, J.L.: Microchannel plate detectors. Nuclear Instruments \& Methods in Physics Research 162, 587-601 (1979)

5. Bateman, J.E., Apsimon, R.J., Barlow, E.E.: A new photomultiplier tube utilising channel palte electron multipliers as the gain producing elements. Nuclear Instruments \& Methods in Physics Research B 137, 61-70 (1976)

6. Lo, C.C., Lecomte, P., Leskovar, B.: Performance studies of prototype microchannel plate photomultipliers. IEEE Transactions on Nuclear Science 24(1), 302-311 (1977)

7. Lo, C.C., Leskovar, B.: Performance of high gain photomultiplier having Z-configuration of microchannel plates. IEEE Transactions on Nuclear Science 28(1), 698-704 (1981) 
8. Edgar, M.L., Kessel, R., Lapington, J.S., Walton, D.M.: Spatial charge cloud distribution of microchannel paltes. The Review of Scientific Instruments 60(12), 3673-3680 (1989)

9. Lapington, J.S.: The effects of secondary electron emission on the operation of position sensitive anodes. Nuclear Instruments \& Methods in Physics Research A 392, 336-340 (1997)

10. Tremsin, A.S., Siegmund, O.H.W.: Spatial distribution of electron cloud footprints from microchannel plates: Measurements and modeling. The Review of Scientific Instruments 70(8), 3282-3288 (1999)

11. Price, G.J., Fraser, G.W.: Calculation of the output charge cloud from a microchannel plate. Nuclear Instruments \& Methods in Physics Research A 474, 188-196 (2001)

12. Oberheide, J., Wilhelms, P., Zimmer, M.: New results on the absolute ion detection efficiencies of a microchannel plate. Measurement Science and Technology 8, 351-354 (1997)

13. Deconihout, B., Vurpillot, F., Bouet, M.: Improved ion detection efficiency of microchannel plate detectors. The Review of Scientific Instruments 73(4), 1734-1740 (2002)

14. Fraser, G.W.: The ion detection efficiency of microchannel plates (MCPs). International Journal of Mass Spectrometry 215(1-3), 13-30 (2002)

15. Morenzoni, E., Oba, K., Pedroni, E., Taqqu, D.: Performance of microchannel plates in high magnetic fields. Nuclear Instruments \& Methods in Physics Research A 263, 397-400 (1988)

16. Fraser, G.W.: The gain, temporal resolution and magnetic-field immunity. Nuclear Instruments \& Methods in Physics Research A 291, 595-606 (1990)

17. Ali, I., Dorner, R., Jagutzki, O., Nuttgens, S., Mergel, V., Spielberger, L., Khayyat, K., Vogt, T., Brauning, H., Ullmann, K., Moshammer, R., Ullrich, J., Hagmann, S., Groeneveld, K.O., Cocke, C.L., SchmidtBocking, H.: Multi-hit detector system for complete momentum balance in spectroscopy in molecular fragmentation processes. Nuclear Instruments and Methods in Physics Research Section B: Beam Interactions with Materials and Atoms 149(4), 490-500 (1999)

18. Jagutzki, O., Cerezo, A., Czasch, A., Dorner, R., Hattass, M., Huang, M., Mergel, V., Spillmann, U., Ullmann-Pfleger, K., Weber, T., Schmidt-Bocking, H., Smith, G.D.W.: Multiple hit readout of a microchannel plate detector with a three-layer delay-line anode. IEEE Transactions on Nuclear Science 49(5), 2477-2483 (2002)

19. Spillmann, U., Jagutzki, O., Spielberger, L., Dorner, R., Mergel, V., Ullmann-Pfleger, K., Schmidt-Bocking, H.: A novel delay-line anode design for position and time sensitive read-out of MCP-based detectors. Physica Scripta T92, 225-226 (2001)

20. Riu, J.R., Stankiewicz, M., Karawajczyk, A., Winiarczyk, P.: A position-sensitive time-of-flight analyser for study of molecular photofragmentation. Nuclear Instruments \& Methods in Physics Research A 477, 360-364 (2002)

21. Firmani, C., Ruiz, E., Carlson, C.W., Lampton, M., Paresce, F.: Highresolution imaging with a two-dimensional resistive anode photon counter. The Review of Scientific Instruments 53(5), 570-574 (1982)

22. Downie, P., Litchfield, D., Parsons, R., Reynolds, D.J.: High-resolution position-sensitive resistive anode microchannel palte detector systems suitable for megahertz count-rates. Measurement Science and Technology 4, 1293-1296 (1993)

23. Murakami, G., Yoshioka, K., Yoshikawa, I.: High-resolution imaging detector using five microchannel plates and a resistive anode encoder. Applied Optics 49(16), 2985-2993 (2010)

24. Martin, C., Jelinsky, P., Lampton, M., Malina, R.F., Anger, H.O.: Wedge-and-strip anodes for centroid-finding position-sensitive photon and particle detectors. The Review of Scientific Instruments 52(7), 1067-1074 (1981)

25. Horio, T., Suzuki, T.: Multihit two-dimensional charged-particle imaging system with real-time image processing at 1000 frames/s. The Review of Scientific Instruments 80(1), 1-8 (2009)

26. Kella, D., Algranati, M., Feldman, H., Heber, O., Kovner, H., Malkin, E., Miklazky, E., Naaman, R., Zajfman, J., Vager, Z.: Asystem for Coulomb explosion imaging of small molecules at the Weizmann Institut. Nuclear Instruments \& Methods in Physics Research A 329, 440-452 (1993)

27. Sinha, M.P., Wadsworth, M.: Miniature focal plane mass spectrometer with 1000-pixel modified-CCD detector array for direct ion measurement. The Review of Scientific Instruments 76(2), 1-8 (2005)

28. Sinha, M.P., Neidholdt, E.L., Hurowitz, J., Sturhahn, W., Beard, B., Hecht, M.H.: Laser ablation-miniature mass spectrometer for elemental and isotopic analysis of rocks. The Review of Scientific Instruments $\mathbf{8 2}$ (9), 094102 (2011)

29. Graham, A.W.G., Ray, S.J., Enke, C.G., Barinaga, C.J., Koppenaal, D., Hieftje, G.M.: First Distance-of-Flight Instrument: Opening a New Paradigm in Mass Spectrometry. Journal of the American Society for Mass Spectrometry 22(1), 110-117 (2011) 